\title{
Interaction particles from the surface of the curved pipeline
}

\author{
Michail Vasilevsky ${ }^{1}$, Aleksandr Razva ${ }^{1, *}$, Aleksandr Naumkin $^{1}$, Dmitriy Zaharov ${ }^{1}$ and \\ Eugene Zykov ${ }^{1}$ \\ ${ }^{1}$ National research Tomsk polytechnic university, 634050 Tomsk, Russia
}

\begin{abstract}
The interaction of the agglomerated fine dust particles from the surface of the rotary pyleprovoda, given deposit formation evaluation. The interaction of large particles to the surface of the rotary pyleprovoda. The analysis of hydrodynamic phenomena in the means of protection against wear.
\end{abstract}

\section{Introduction}

The disperse material coming to the dust flue consists of sets of primary particles, the aggregated particles, and represents an aerosol together with the bearing environment. The agglomerates are formed by coagulation turbulent, with flow disrupting deposits from surfaces with suction materials, particle segregation as a result of various transportation devices in [1]. Rotating flow in curved channel leads to the centrifugal inertial force acting across the flow, which in turn changes the conditions of gas motion. There are secondary flow of the first and the second kind [1], whose impact on the separation of the particles is shown, depending on the shape of the channel, the concentration and size of particles [1]. Curvilinear motion of the dust-laden stream is accompanied by interaction with the surface of the particles, if their speed due to centrifugal force is much greater than the turbulent transfer rate near the surface [1]. In [2] shows the calculated distribution model particle concentrations that are not deposited on the wall due to turbulent eddies. It is assumed that the total flux of particles due to centrifugal forces and turbulent diffusion transfer should be zero.

The agglomerates are composed of many particles, so have a lot of large compared to the mass of the individual particles. Depending on the ratio of the radial and tangential velocity of sinter approaching it to the surface is carried out at different angles (Figure 1).

Large particles (greater than 20 microns) are abrasives. The intensity of abrasive wear of the apparatus depends on the hardness, size, and particle density. Causes of wear: impact action of abrasive particles, particles of metal cutting, fatigue accumulation in the surface layer of the wear material, heat and fatigue failure after repeated collision particles. As a result of repeated plastic deformation of the material causing the residual metal lattice distortion, the process of the fracture surface.

\footnotetext{
* Corresponding author: razva@mail.ru
} 


\section{The calculated ratio of the interaction with the surface of the agglomerate}

The hade defines the nature of its interaction with a surface. Agglomerate density is several times lower compared to the density of the particulate material of which it consists. Therefore ricocheting hardly possible. Depending on the angle and speed of interaction of interaction will agglomerate or blurred, and again dispersed to coarsen, or vice versa, will condense and form deposits. If deposits are strong, they do not break the flow. Figure 1 shows the motion trajectory of the agglomerate in the channel conversion circuit agglomerate A (dashed line) in the sealed clot B. Agglomerates composed of fine particles under the influence of centrifugal force moves relative to the periphery of the gas and the interaction with the sealing wall extends stage deformation aftereffect.

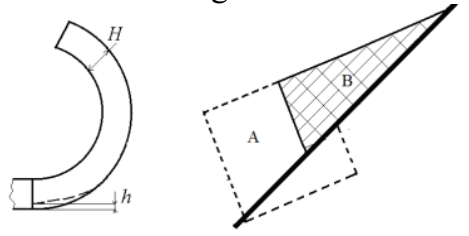

Fig. 1. Trajectories of the movement of agglomerate in the port and the scheme of transformation of agglomerate $\mathrm{A}$ (dotted line) in the condensed clot B.

Changing the amount of movement is equal to the impulse force generated in the formed contact surface. Furthermore, it can be used the energy change rate equation relating the kinetic energy and rate of change of energy of the peripheral forces applied to the deformable surface. All these data allow us to predict the status and parameters of sinter, if you know the parameters of the particle layer deposition.

The particle speed relative to the gas stream in a curvilinear radial direction can be determined from the dimensionless equation [1]

$$
\begin{gathered}
\frac{\overline{W_{\phi}^{2}}}{\bar{r}}=\Psi\left(\operatorname{Re}_{\delta}\right) \frac{\operatorname{Re}_{\delta}{ }^{2}}{24 S t k \cdot \operatorname{Re}_{\delta, w}}, \\
\operatorname{Re}_{\delta, w}=\frac{W_{0} \delta}{v}, S t k=\frac{\delta^{2}}{18 v} \frac{\rho_{\delta}}{\rho} \frac{W_{0}}{R_{c}}, S t k \cdot \operatorname{Re}_{\delta, w}=\frac{\rho_{\delta}}{\rho} \frac{W_{0}^{2}}{R_{c}} \frac{\delta^{3}}{18 v^{2}}, \operatorname{Re}_{\delta}=\frac{\Delta U \delta}{v},
\end{gathered}
$$

where $\rho, \rho_{\delta}-$ gas and particle density, $\delta$ - particle diameter, $v$ - kinematic speed gas, $\Delta U$ - particles relative to the gas speed in the radial direction, $\mathrm{W}_{0}-$ gas speed at the inlet of the apparatus, $\overline{W_{\phi}}=\frac{W_{\phi}}{W_{0}}-$ circumferential dimensionless particle speed, $\mathrm{R}_{\mathrm{c}}-$ base value turning radius.

Agglomerates near a wall pass the site with the reducing stream speed, and on the wall the speed of a stream is equal to zero. District speed of gas at distance from a wall [3]:

$$
W_{\phi}(y)=\sqrt{\frac{\tau_{w}}{\rho}}(9.5+1.32 \ln \eta), \quad \eta=\frac{y}{v} \sqrt{\frac{\tau_{W}}{\rho}}, \quad \tau_{W}=0.5 \mathrm{C}_{f} \rho \mathrm{W}_{\Delta}^{2} / 2
$$

where $C_{f} \approx 0.006, W_{\Delta}$ - the value of the circumferential speed of the wall on the border zone, $W_{\Delta}=0.95 W_{0}$. 
Assume that half the wall of intensive speed $\Delta \approx 0.03 R$ drop zone in the agglomerate does not affect the flow of force is interested, and this zone sinter passes through inertia. We will estimate the value of finite speed of approach of the agglomerate to the wall. Let $x$ $=R-y$, speed of approach to the wall zone $\Delta U_{\Delta}$, speed the fall of the wall in the normal direction $\Delta \mathrm{U}_{\mathrm{st}}$.

The equation of a decelerated motion of agglomerate in the radial direction has an appearance:

$$
m \frac{d \Delta U}{d t}=\xi \frac{\pi \delta^{2}}{4} \frac{\Delta U^{2}}{2} \rho ; \quad m=\frac{\pi \delta^{3}}{6} \rho_{\delta} ; \quad \Delta U=\frac{d x}{d t} ; \quad \frac{d \Delta U}{d t}=\frac{d \Delta U^{2}}{2 d x}
$$

After integration, we will receive the following expression

$$
\frac{\Delta U_{c m}}{\Delta U_{\Delta}}=\sqrt{\exp \left(-\frac{3}{2} \xi \frac{\rho}{\rho_{\delta}} \frac{\Delta}{2 \delta}\right)}
$$

Let's consider the simplest process of sealing of agglomerate at its normal contact interaction with a barrier. Speed of blow of $V_{0}$ is directed on a cylinder shaft perpendicular to the barrier plane. Process of blow is followed by compression of the disperse environment with small change of cross-sectional area. This can happen in the case of interaction of the particles within the cylinder, in which each contact is inelastic, and the particles are attracted to each other autogezionny forces. This is typical of a size less than 10 microns. The initial length $L_{1}$ of the cylinder, the length $L_{2}$ in the compressed state (figure 2).

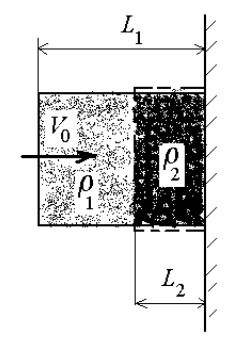

Fig. 2. Schematic of the interaction with the surface of the cylindrical sinter at a right angle.

At blow the disperse environment from volume with a length of $L_{1}-L_{2}$ passes into volume with $L_{2}$ length. Let's put that before blow and after blow firmness of the environment in volumes is distributed evenly. The condition of preservation of mass of the disperse environment in position of cylinders 1 and 2 gives the following ratio:

$$
\rho 1 \mathrm{~L} 1=\rho 2 \mathrm{~L} 2
$$

The equation of impulses at interaction of agglomerate with a wall has an appearance

$$
\rho_{1} L_{1} \frac{d V}{d t}=-\sigma_{s} ; \quad d t=\frac{d l}{V_{0}},
$$

Where $t$ - variable time, $l$ - the variable length, $V$ - variable speed, $\sigma_{s}$ - contact piece tension on a wall.

The last equation can be written in difference form:

$$
\rho_{1} L_{1} \frac{\Delta V_{0}^{2}}{\Delta l}=-\sigma_{s}
$$

Sharing and taking into account factors that $\Delta l=L_{l}-L_{2}$, using equation (3), we obtain the relation between the density, speed and voltage: 


$$
\sigma_{s}=V_{0}^{2} \frac{\rho_{1}}{1-\frac{\rho_{1}}{\rho_{2}}},
$$

Firmness of agglomerate defines its strength indicators which, in turn, are defined by autogezionny internal forces. From equation (4), that the contact voltage, which are under the above assumptions and the voltage in the agglomerate at the end of the contact interaction, determined by the density and speed to agglomerate and the density of impact after impact. However, it is clear that tension in agglomerate has to decrease to a running end therefore tension, average on length, has to be less, than tension at a butt.

Represent the distribution of stress over the length of a volume with a length $L_{2}$ :

$$
\sigma=\sigma_{s}\left(1-\frac{l}{L_{2}}\right)^{k},
$$

After the termination of influence of all loadings (the after-effect period) agglomerate in a new state appears in balance. Agglomerate is affected by the carrying-away aerodynamic forces from a stream, gravity, on the other side of - the wall force attracting due to adhesion and an autogeziya of particles. In a new state at a free butt there is no pressure force whereas adhesive forces act on a contact butt.

The formula (3) and formulas (4) and (5) allow to carry out calculation of contact tension and to estimate distribution of tension in agglomerate during interaction it with a wall. Calculation is carried out for two values of the relations of firmness $\rho 1 / \rho 2=0,7$ and $\rho 1 / \rho 2=0,4, \rho 1=300$ by $\mathrm{kg} / \mathrm{m}^{3}$. In a formula (2) $\Delta U c_{m} \equiv V_{0}$.

Calculation of tension in the agglomerate on a formula (5) it shows the rapid reduction of tension to a running end.

Thus, information on firmness of deposits can be also information on the speed of approach of agglomerate to a surface and about its weight. Estimates show that tension in interactions of agglomerate with a surface can reach the values comparable to atmospheric pressure that is the reason of formation of strong deposits.

\section{Hydrodynamic effects in anti-devices}

It was found that the intensity of abrasive wear is proportional to the third or fourth degree of speed of particles at the time of blow [4]. For reduction of wear of a wall of the dust flue by its surfaces, carry out pockets or fins (Figure 3) [5].

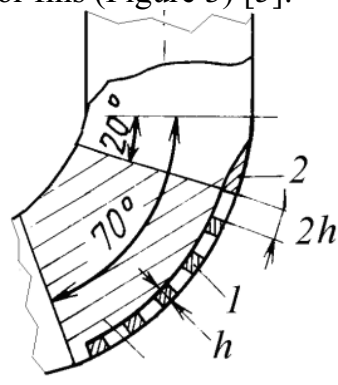

Fig. 3. The circuit surface protection rotary dust flue wear: 1 - rib height $h ; 2-$ configuration of the first rib to the unstressed entering the oncoming flow.

When passing a stream in a branch pipe of a particle of ashes deviate to its external ribbed wall. The large-scale whirlwinds arising near a concave are split up by edges for 
smaller vortex educations which scale is proportional to the cell size between edges. In lack of large-scale turbulent transfer the particles weighed in a stream on the whole can't reach a wall of the rotary gas flue. Speed of those particles that hit against a gas flue wall, considerably decreases in the presence of edges owing to braking of a stream, formation of aerodynamically shadow zones and stagnant currents of small scale in intercostal space [5].

The intensity of the induced motion of the gas in the intercostal region can be estimated [6], where it obtained the hydrodynamic flow pattern of a viscous liquid in an open cavity in conditions of forced convection and studied the influence of the model parameters on the character of the movement. After contact with the fine particles in the cavity between the ribs and variations are possible deposition cavity. In this case, you can use the results of [7], in which the numerical simulation of heat transfer and hydrodynamics in motion of a viscous incompressible non-isothermal liquid in an open rectangular cavity in view of the melting process of its walls. With intensive heat removal motion of the gas in the cavity is calculated using the results of [8], which studied the effect of model parameters on the nature of the flow and the cooling cavity.

Results of tests of rotary dust flues with fins showed essential reduction of wear of surfaces at invariable hydraulic resistance [5]. Test results from the rotary dust pipe fins showed a significant reduction in wear surface at a constant flow resistance [5]. [9] The calculation of the abrasive wear of pipelines and pneumatic transport systems of TPP ash coal dust, [10] provides recommendations for optimizing the parameters of piping bends his knees pneumatic ash handling systems and installations pulverization TPP.

\section{Conclusion}

To improve the reliability of dust systems must take into account the properties of dust particle flows, with the possible prediction of possible deposits on turning dust pipe areas, and at high content of large abrasive particles to take measures for the protection of surfaces from wear.

\section{References}

1. M. V. Vasilevsky, Dust removal of gases inertial devices 248 (Tomsk, TPU, 2008)

2. M. V. Vasilevsky, E.G. Zykov, and A. S. Razva, Theor. Found. Chem. Eng. 45, 321 (2011)

3. A. N. Shtym, Aerodynamics of cyclonic vortex cameras (Far Eastern University, Vladivostok, 1985)

4. J. A. Tadolder, On wear of metals at high jet speeds 83 (Works of the Tallinn polytechnic institute, 1975)

5. V. A. Reznik, N. N. Prokofiev, E. L. Alexandrov, Turning the boiler dust pipeline (patent Russian № 2059932 10.05.1996)

6. G. V. Kuznetsov, A. V. Krainov, J. Appl. Mech. Techn. Phys. 42, 851 (2001)

7. G. V. Kuznetsov, A. V. Krainov, J. High Temp. 41, 252 (2003)

8. G. V. Kuznetsov, A. V. Krainov, EPJ Web of Conf. 76, 01023 (2014)

9. V. Y. Putilov, I. V. Putilova, Therm. Eng. 9, 60 (2003)

10. V. Y. Putilov, I. V. Putilova, Energy saving and water treatment, 3, 67 (2004) 